\title{
Vedolizumab en tratamiento de colitis ulcerativa refractaria asociada con psoriasis: reporte de caso
}

\section{Case Report of Vedolizumab Treatment of Refractory Ulcerative Colitis Associated with Psoriasis}

Gabriel Alonso Mosquera-Klinger, ${ }^{1 *}$ María Alejandra Zambrano-Gustín, ${ }^{2}$ Jhon Jaime Carvajal. ${ }^{1}$

\footnotetext{
Especialista en medicina interna, gastroenterología y endoscopia digestiva, Unidad de Gastroenterología, Hospital Pablo Tobón Uribe. Medellín, Colombia.

2 Médica, Pontificia Universidad Javeriana de Cali. Cali, Colombia.

*Correspondencia: gami8203@yahoo.com.

Fecha recibido: $\quad 30 / 01 / 18$

Fecha aceptado: $25 / 03 / 18$
}

\begin{abstract}
Resumen
Paciente con historia de psoriasis de difícil control y colitis ulcerativa de larga evolución, corticodependiente, requirió múltiples esquemas de tratamientos incluidos varios antagonistas de factor de necrosis tumoral (antiTNF), con lo cual tuvo respuesta clínica aceptable y posterior pérdida de efectividad. En una exacerbación aguda de la colitis se utilizó vedolizumab, con lo cual se logró una notable mejoría clínica, y en el mantenimiento se llegó a cicatrización profunda (normalización endoscópica e histológica), además de mejoría significativa de las lesiones de psoriasis en el seguimiento a 12 meses.

En lo conocido hasta ahora, es el primer caso con el uso de vedolizumab en esta indicación publicado en Colombia.
\end{abstract}

Palabras clave

Terapia biológica, psoriasis, colitis ulcerativa, vedolizumab.

\begin{abstract}
The patient had a long history of difficult to control psoriasis and ulcerative colitis and was dependent on corticosteroids. Multiple treatment schemes including several attempts with tumor necrosis factor (anti-TNF) antagonists had had acceptable clinical responses but subsequently lost effectiveness. During acute exacerbation of colitis, vedolizumab was used and achieved remarkable clinical improvement. During maintenance, deep healing was achieved (endoscopic and histological normalization). In addition, there was significant improvement of psoriasis lesions during follow-up.

This is the first case to be published of the use of Vedolizumab to treat this condition in Colombia.
\end{abstract}

Keywords

Biological therapy, psoriasis, ulcerative colitis, vedolizumab.

\section{INTRODUCCIÓN}

La enfermedad inflamatoria intestinal (EII) comprende la enfermedad de Crohn (EC), la colitis ulcerativa (CU) y la colitis indeterminada (este último término en la actualidad se considera reservado para patólogos cuando tienen el espécimen de una colectomía en pacientes con hallazgos sobrepuestos o no definitorios entre EC y CU) (1).
La etiología y la patogénesis exacta aún se desconocen, pero la hipótesis actual relaciona a la EII con la desregulación del sistema inmune gastrointestinal en individuos genéticamente predispuestos (2). Algunos autores mencionan la importancia de vías patógenas como interleucina 23 (IL-23), que media la defensa microbiana y la inflamación intestinal; y genes como el receptor de IL-23 (IL-23R) e interleucina 12B (IL-12B) se han asociado $(3,4)$. Estos 
genes y otros como el de la proteína tirosina-cinasa 2 (TYK2), la cinasa Janus 2 (JAK2), el transductor de señal y el activador de la transcripción 3 (STAT3) también están asociados con psoriasis (2).

La incidencia de EC y CU es 3,8 a 7,5 veces mayor en pacientes con psoriasis que en la población general (5). En ambas patologías, los anticuerpos antifactor de necrosis tumoral alfa (anti-TNF $\alpha$ ) como el infliximab y el adalimumab tienen un efecto benéfico, lo que respalda la hipótesis de vías inflamatorias comunes dirigidas tanto a la piel como al intestino. La EII y la psoriasis podrían superponerse, o incluso la psoriasis se podría presentar de forma paradójica en personas que hayan recibido anti-TNF para EII. (se estima que las lesiones psoriasiformes en la piel podrían ocurrir en 1,62 \%-8,8 \% de los pacientes con EII tratados con anti-TNF) $(6,7)$. La aparición de lesiones psoriasiformes no está asociada con el sexo, duración de tratamiento o actividad de la EII (8-10). En muchos individuos, el control de la EII podría impactar positivamente en la mejoría o resolución de lesiones de la psoriasis.

La CU es la forma más común de EII. En el estudio realizado en el Hospital Pablo Tobón Uribe (HPTU) en Medellín, Colombia, la relación entre CU y EC fue 4,9:1 (CU en 80,7 \%, EC en 15,8\%) (11). La incidencia de CU es variable: según la población estudiada, entre 1,2 a 20,3 casos por cada 100000 personas/año (7). La etiología es desconocida y multifactorial; se ha descrito que la historia familiar de EII incrementa el riesgo; la apendicectomía en jóvenes, así como el hábito de fumar podrían ser factores protectores
$(1,12)$. La CU se caracteriza por la presencia de inflamación crónica y difusa en la mucosa rectal y colónica (1). En el curso natural de la enfermedad alternan frecuentemente brotes de actividad inflamatoria con períodos de remisión y existe una elevada tendencia a la recurrencia (13).

\section{CASO CLÍNICO}

Una paciente de 50 años, con historia personal de CU (diagnóstico en el 2009) y psoriasis (diagnóstico en 1996) fue conocida en el HPTU en agosto de 2016 por cuadro de dolor anal y disquecia de al menos 2 meses de evolución, asociados con diarrea acuosa, sangre y, en la última semana, exacerbación de sus síntomas, teniendo en promedio 10-12 deposiciones al día; y dolor abdominal tipo cólico a diario. En la revisión por sistemas refirió intenso prurito y rascado frecuente de las lesiones en la piel. En el examen físico se notó con palidez mucocutánea; deshidratada; afebril; con dolor abdominal sin irritación peritoneal; con múltiples placas descamativas activas en cara, tronco y extremidades; múltiples máculas de hiperpigmentación y erosiones de rascado en la piel (Figura 1 A y B). Se hospitalizó y se solicitaron estudios de ingreso (Tabla 1), en los que se identificó anemia leve y marcada elevación de reactantes de fase aguda. En la colonoscopia solo se llegó hasta el ángulo esplénico por severos cambios inflamatorios y sangrado fácil con el roce del equipo, y por el alto riesgo de perforación se decidió suspender a ese nivel (Figura 2). Se solicitaron exámenes para descartar procesos infecciosos sobreagregados (toxina
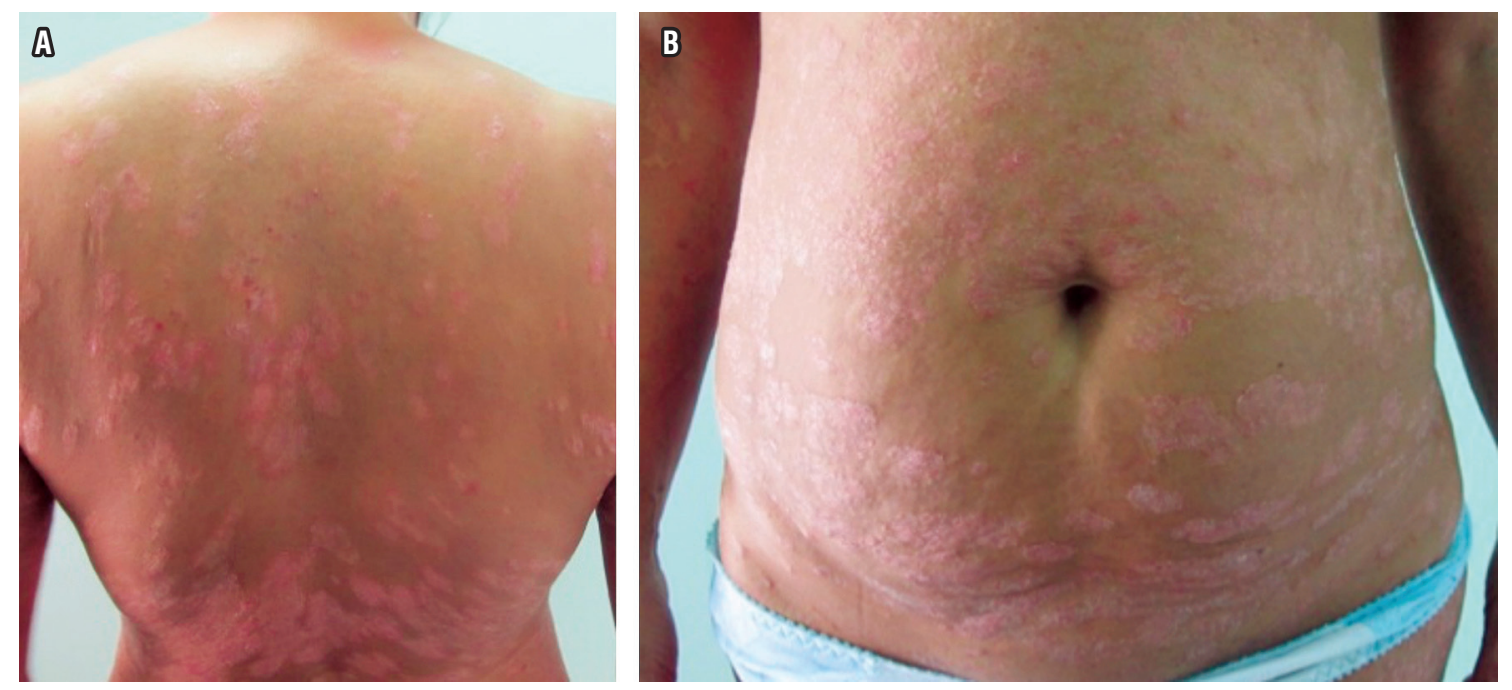

Figura 1. A. Foto del dorso de la paciente, donde se evidencian múltiples lesiones descamativas, eritematoedematosas, con erosiones de rascado. B. Foto de la piel del abdomen, donde se observan múltiples placas eritematosas, descamativas, con erosiones de rascado. 
Tabla 1. Exámenes durante la hospitalización

\begin{tabular}{|c|c|c|c|c|c|}
\hline Exámenes solicitados & $\begin{array}{c}\text { Exámenes de } \\
\text { ingreso }(12 / 08 / 16)\end{array}$ & $15 / 08 / 16$ & $19 / 08 / 16$ & $23 / 08 / 16$ & $\begin{array}{l}\text { Valores de } \\
\text { referencia }\end{array}$ \\
\hline Hemoglobina & $11,6 \mathrm{~g} / \mathrm{dL}$ & $9,4 \mathrm{~g} / \mathrm{dL}$ & $9,4 \mathrm{~g} / \mathrm{dL}$ & $9,3 \mathrm{~g} / \mathrm{dL}$ & $12-15 \mathrm{~g} / \mathrm{dL}$ \\
\hline Hematocrito & $36,3 \%$ & $28,9 \%$ & $29,3 \%$ & $28,5 \%$ & $36 \%-46 \%$ \\
\hline Leucocitos & $16200 \mathrm{~mm}^{3}$ & $17700 \mathrm{~mm}^{3}$ & $22900 \mathrm{~mm}^{3}$ & $26200 \mathrm{~mm}^{3}$ & $4500-11000 \mathrm{~mm}^{3}$ \\
\hline Plaquetas & 593000 & 483000 & 524000 & 497000 & $150000-450000$ \\
\hline VSG & $100 \mathrm{~mm} / \mathrm{h}$ & & $77 \mathrm{~mm} / \mathrm{h}$ & $72 \mathrm{~mm} / \mathrm{h}$ & $1-20 \mathrm{~mm} / \mathrm{h}$ \\
\hline Clostridium: toxinas A y B & Negativo & & & & Negativo \\
\hline PCR & & $4,56 \mathrm{mg} / \mathrm{dL}$ & $3,04 \mathrm{mg} / \mathrm{dL}$ & $0,93 \mathrm{mg} / \mathrm{dL}$ & $0,01-0,82 \mathrm{mg} / \mathrm{dL}$ \\
\hline Hierro & & $57 \mu \mathrm{g} / \mathrm{mL}$ & & & $60-170 \mu \mathrm{g} / \mathrm{mL}$ \\
\hline Ferritina & & $35,2 \mathrm{ng} / \mathrm{mL}$ & & & $15-150 \mathrm{ng} / \mathrm{mL}$ \\
\hline
\end{tabular}

VSG: velocidad de sedimentación globular.
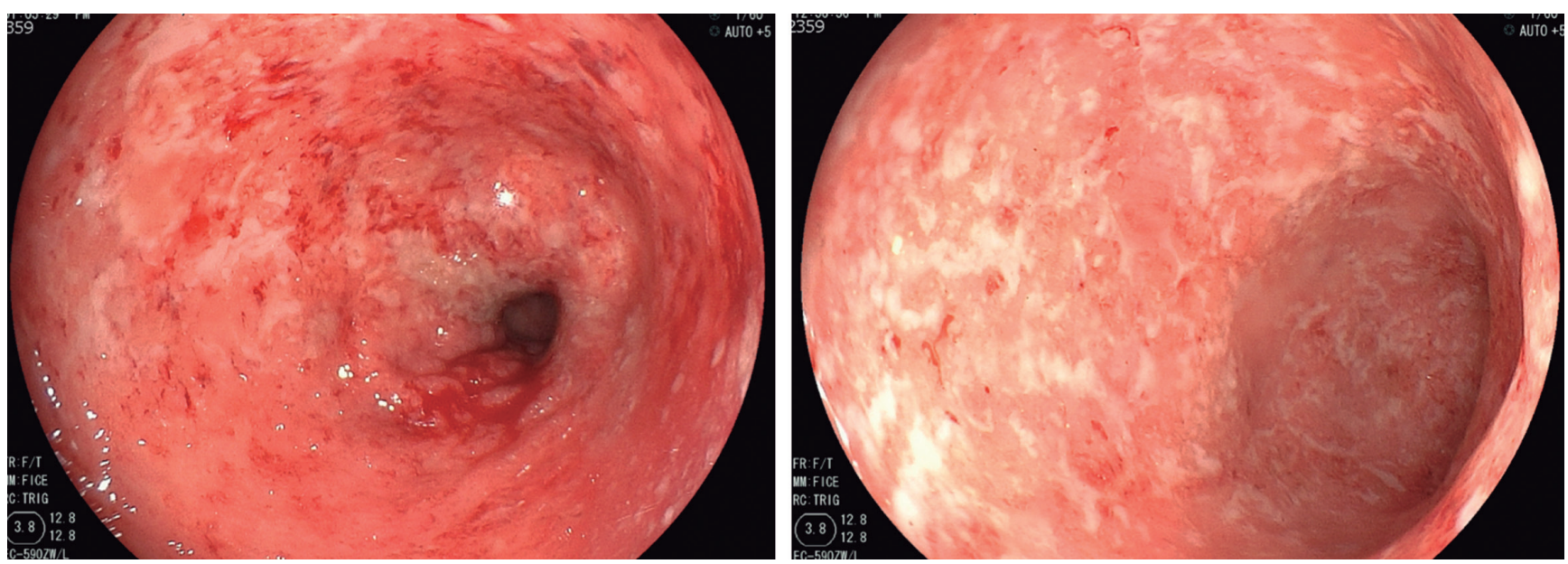

Figura 2. Colonoscopia donde se evidencian severos cambios inflamatorios, dados por la pérdida del patrón vascular normal. Marcado edema, eritema, erosiones múltiples homogéneas y mucosa friable al roce.

A y B para Clostridium negativa, inmunohistoquímica de citomegalovirus [CMV] en el tejido negativa). Inicialmente, se manejó con hidrocortisona $100 \mathrm{mg}$ cada 8 horas durante 5 días, mesalazina oral en gránulos $1 \mathrm{~g} / 8 \mathrm{~h}$ y mesalazina en enemas $4 \mathrm{~g} /$ día.

Adicionalmente, se revisó la historia clínica extrainstitucional para conocer al detalle el tratamiento previo, en la cual se describían múltiples esquemas recibidos: mesalazina en gránulos $3 \mathrm{~g} /$ día, azatioprina $100 \mathrm{mg} /$ día, hierro parenteral quincenal por anemia e infliximab en dosis de $5 \mathrm{mg} / \mathrm{kg}$ durante 5 años; durante el último año de uso del infliximab perdió efectividad, por lo que requirió aumentar la dosis hasta $10 \mathrm{mg} / \mathrm{kg}$ sin una adecuada respuesta; luego cambian por adalimumab a dosis de $40 \mathrm{mg}$ cada 2 semanas (2014-2015). Con el adalimumab mantenía una frecuen- cia defecatoria promedio entre 3-6 deposiciones al día, sin sangre, pero a finales de 2015 tuvo una nueva exacerbación y empeoramiento de las lesiones en la piel, por lo que se cambió el manejo a ustekinumab $45 \mathrm{mg}$ subcutáneo (SC) cada 2 meses (2015-2016). Con este último hubo mínima mejoría de los síntomas digestivos, pero hubo un pobre control de las lesiones en la piel. Por recomendación de dermatología se cambió a secukinumab en marzo de 2016 y se continuó con mesalazina y azatioprina hasta la fecha de ingreso a nuestra institución. Con este último tratamiento presentaba una pobre respuesta de las manifestaciones gastrointestinales, por lo cual se hospitalizó.

Durante la hospitalización se observó un alto gasto fecal (con deposiciones sanguinolentas entre 6-7 diarias), anemización progresiva y persistencia de reactantes de fase 
aguda elevados (Tabla 1). Se realizó un staff de gastroenterología, donde se definió iniciar vedolizumab con esquema de inducción de $300 \mathrm{mg}$ en la semana 0 , semana 2 , semana 6 y luego cada 8 semanas.

Durante el seguimiento ambulatorio la paciente refirió una notable mejoría, llegando a estar asintomática 3 meses después de iniciado el tratamiento. Se observó la normalización de los reactantes de fase aguda y normalización de la hemoglobina sin requerir administración de hierro parenteral. Además, se suspendió la azatioprina y se adicionó metotrexato a $12,5 \mathrm{mg} /$ semana por su mejor perfil sobre el compromiso dermatológico.

En los controles endoscópicos se evidenció normalización de la mucosa, con biopsias histológicamente normales. Refirió, además, control completo del prurito y no ha requerido más uso de esteroides tópicos.

En el seguimiento a 12 meses ha estado asintomática desde el punto de vista digestivo, en remisión profunda y sin evidencia de actividad de sus lesiones de psoriasis. Se programó para exámenes de seguimiento cada 6 meses y evaluación clínica entre 3-6 meses.

\section{DISCUSIÓN}

El tratamiento de la EII se ha basado principalmente en la estrategia step-up. En CU se considera apropiado el manejo inicial con 5-aminosalicilatos (5-ASA) para CU leve, tanto para inducción de remisión, como para el mantenimiento. En pacientes con enfermedad moderada-severa o leves en quienes falle el tratamiento inicial con 5-ASA está indicado el uso de cursos cortos (entre 2-4 meses) con esteroides. Los pacientes corticodependientes o quienes tengan recurrencias o pérdida de eficacia con el glucocorticoide son candidatos al uso de inmunomoduladores como las tiopurinas (azatioprina, 6-mercaptopurina) y el metotrexato (14); y en individuos corticorresistentes (que no responden a esteroides) o corticodependientes están indicados los anti-TNF como el infliximab, adalimumab, golimumab o certolizumab (15). El problema radica en que un número considerable de pacientes no tiene una respuesta clínica significativa (falla primaria al tratamiento), este fenómeno ocurre entre $10 \%-40 \%$ de casos de EC y en casi el $50 \%$ de los pacientes con CU (15-18); adicionalmente, se describe en especial en EC que hasta 2 tercios de pacientes tienen falla secundaria al tratamiento (falta de respuesta sostenida durante 12 meses de tratamiento continuo) $(14,19,20)$. En los casos de persistencia de la inflamación a pesar de tratamiento óptimo (dosis estándar de anti-TNF y del resto de medicamentos) hay clara indicación para aumentar la dosis o acortar el intervalo de aplicación del anti-TNF (15), como se hizo en esta paciente.

Para la CU en la actualidad se están utilizando nuevos esquemas de tratamiento con mecanismos de acción diferentes que parecen prometedores en pacientes refractarios a los anti-TNF. El vedolizumab es un anticuerpo antiintegrina $\alpha_{4} \beta_{7}$ selectivo en el tracto gastrointestinal, con evidencia creciente en relación con el tratamiento de pacientes con EII, especialmente en CU $(1,12,21,22)$. Incluso se ha usado en casos de exantema psoriasiforme asociado con el uso de anti-TNF (23). Este medicamento fue aprobado
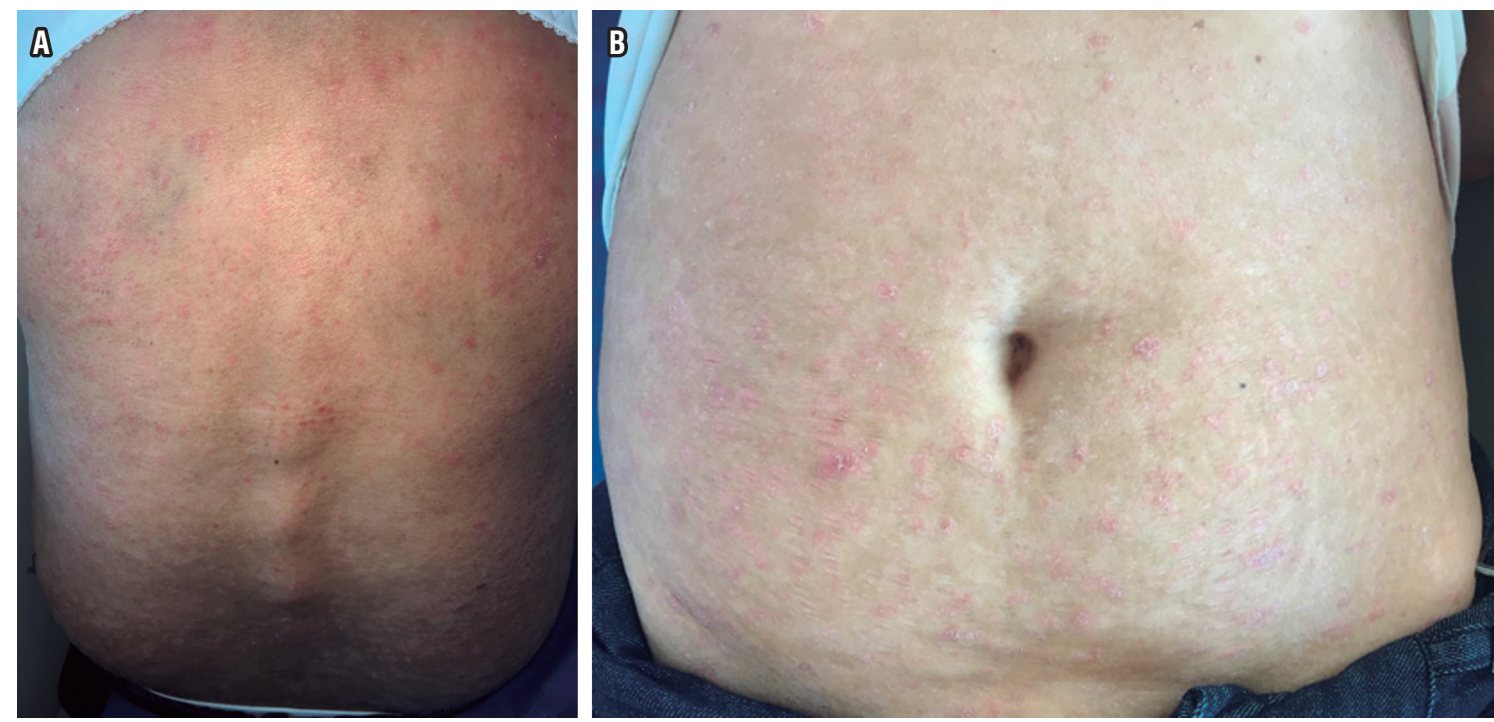

Figura 3. A. Foto del dorso del paciente a 12 meses de seguimiento. B. Piel del abdomen, control en 12 meses de seguimiento. 
por el Instituto Nacional de Vigilancia de Medicamentos y Alimentos (INVIMA) en febrero de 2016 para el uso en Colombia en EII, en pacientes adultos con enfermedad activa moderada-severa, que hayan tenido una respuesta inadecuada, presenten pérdida progresiva o sean intolerantes a los esteroides, inmunomoduladores o anti-TNF $\alpha$.

A pesar de no tener suficiente evidencia científica en psoriasis, decidimos utilizarlo en esta paciente ya que había un claro predominio de manifestaciones gastrointestinales, que en este punto podrían llevarla a complicaciones mayores como sangrado severo, anemia, necesidad de transfusión de hemoderivados, megacolon tóxico, perforación e incluso hasta la muerte. Además, ya se había agotado el manejo médico disponible en nuestro medio para su tratamiento. Con esta intervención logramos el control de ambas patologías por la evidencia de cicatrización profunda de la mucosa colónica y control de la psoriasis en el seguimiento a 12 meses (Figura 3 A y B). La mejoría notable en las lesiones de piel fue una ganancia clínica adicional que ha impactado de forma muy positiva en la calidad de vida de la paciente. Consideramos que se requieren serie de casos y estudios controlados para evaluar el impacto del medicamento sobre las lesiones de psoriasis. En lo revisado hasta hoy, este es el primer caso publicado de vedolizumab en un paciente colombiano con CU refractaria y psoriasis.

\section{REFERENCIAS}

1. Magro F, Gionchetti P, Eliakim R, Ardizzone S, Armuzzi A, Barreiro-de Acosta M, et al. Third European Evidencebased Consensus on Diagnosis and Management of Ulcerative Colitis. Part 1: Definitions, Diagnosis, Extraintestinal Manifestations, Pregnancy, Cancer Surveillance, Surgery, and Ileo-anal Pouch Disorders. J Crohns Colitis. 2017;11(6):649-670. doi: 10.1093/ecco-jcc/jjx008.

2. Abraham $\mathrm{C}$, Cho JH. Inflammatory bowel disease. $\mathrm{N}$ Engl J Med. 200919;361(21):2066-78. doi: 10.1056/ NEJMra0804647.

3. Duerr RH, Taylor KD, Brant SR, Rioux JD, Silverberg MS, Daly MJ, et al. A genome-wide association study identifies IL23R as an inflammatory bowel disease gene. Science. 2006;314(5804):1461-3.10.1126/science.1135245.

4. Ellinghaus D, Ellinghaus E, Nair RP, Stuart PE, Esko T, Metspalu A, et al. Combined analysis of genome-wide association studies for Crohn disease and psoriasis identifies seven shared susceptibility loci. Am J Hum Genet. 2012;90(4):636-47. doi: 10.1016/j.ajhg.2012.02.020.

5. Najarian DJ, Gottlieb AB. Connections between psoriasis and Crohn's disease. J Am Acad Dermatol. 2003;48(6):805$2110.1067 / \mathrm{mjd} .2003 .540$.

6. Cleynen I, Van Moekercke W, Juergens M, Ballet V, Drobne $\mathrm{D}$, Vandecandelaere $\mathrm{P}$, et al. Anti-TNF induced cutaneous lesions in IBD patients: Characterization and search for predisposing factors. Gut. 2010;59(suppl 3):A1.
7. Afzali A, Wheat CL, Hu JK, Olerud JE, Lee SD. The association of psoriasiform rash with anti-tumor necrosis factor (anti-TNF) therapy in inflammatory bowel disease: a single academic center case series. J Crohns Colitis. 2014;8(6):480-8. doi: 10.1016/j.crohns.2013.10.013.

8. Cullen G, Kroshinsky D, Cheifetz AS, Korzenik JR. Psoriasis associated with anti-tumour necrosis factor therapy in inflammatory bowel disease: a new series and a review of 120 cases from the literature. Aliment Pharmacol Ther. 2011;34(11-12):1318-27. doi: 10.1111/j.13652036.2011.04866.x.

9. Fiorino G, Allez M, Malesci A, Danese S. Review article: anti TNF-alpha induced psoriasis in patients with inflammatory bowel disease. Aliment Pharmacol Ther. 2009;29(9):921-7. doi: 10.1111/j.1365-2036.2009.03955.x.

10. Rahier JF, Buche S, Peyrin-Biroulet L, Bouhnik Y, Duclos B, Louis E, et al. Severe skin lesions cause patients with inflammatory bowel disease to discontinue anti-tumor necrosis factor therapy. Clin Gastroenterol Hepatol. 2010;8(12):104855. doi: 10.1016/j.cgh.2010.07.022.

11. Juliao F, Ruiz M, Flórez J, Donado J, Marín J, Arango C, et al. Fenotipo e historia natural de la enfermedad inflamatoria intestinal en un centro de referencia en Medellín-Colombia. Rev Col Gastroenterol. 2010;25(3):240-51.

12. Yamamoto-Furusho JK, Bosques-Padilla F, de-Paula J, Galiano MT, Ibañez P, Juliao F, et al. Diagnosis and treatment of inflammatory bowel disease: First Latin American Consensus of the Pan American Crohn's and Colitis Organisation. Rev Gastroenterol Mex. 2017;82(1):46-84. doi: 10.1016/j.rgmx.2016.07.003.

13. Marín-Jiménez I, Menchén-Viso L, Gomollón-García F. Diagnóstico diferencial de la enfermedad inflamatoria intestinal. Elsevier; 2012.

14. Nielsen OH, Bjerrum JT, Herfarth H, Rogler G. Recent advances using immunomodulators for inflammatory bowel disease. J Clin Pharmacol. 2013;53(6):575-88. doi: 10.1002/jcph.2.

15. Nielsen OH, Ainsworth MA. Tumor necrosis factor inhibitors for inflammatory bowel disease. N Engl J Med. 2013;369(8):754-62. doi: 10.1056/NEJMct1209614.

16. Rutgeerts P, Sandborn WJ, Feagan BG, Reinisch W, Olson A, Johanns J, et al. Infliximab for induction and maintenance therapy for ulcerative colitis. N Engl J Med. 2005;353(23):2462-76. doi: 10.1056/NEJMoa050516.

17. Reinisch W, Sandborn WJ, Hommes DW, D'Haens G, Hanauer S, Schreiber S, et al. Adalimumab for induction of clinical remission in moderately to severely active ulcerative colitis: results of a randomised controlled trial. Gut. 2011;60(6):780-7. doi: 10.1136/gut.2010.221127.

18. Sandborn WJ, van Assche G, Reinisch W, Colombel JF, D'Haens G, Wolf DC, et al. Adalimumab induces and maintains clinical remission in patients with moderate-to-severe ulcerative colitis. Gastroenterology. 2012;142(2):257-65. e1-3. doi: 10.1053/j.gastro.2011.10.032.

19. Colombel JF, Sandborn WJ, Rutgeerts P, Enns R, Hanauer $\mathrm{SB}$, Panaccione R, et al. Adalimumab for maintenance of cli- 
nical response and remission in patients with Crohn's disease: the CHARM trial. Gastroenterology. 2007;132(1):52-65. doi: 10.1053/j.gastro.2006.11.041.

20. Sands BE, Anderson FH, Bernstein CN, Chey WY, Feagan $\mathrm{BG}$, Fedorak RN, et al. Infliximab maintenance therapy for fistulizing Crohn's disease. N Engl J Med. 2004;350(9):87685. doi: 10.1056/NEJMoa030815.

21. Garnock-Jones KP. Vedolizumab: a review of its use in adult patients with moderately to severely active ulcerative colitis or Crohn's disease. BioDrugs. 2015;29(1):57-67. doi: $10.1007 / \mathrm{s} 40259-014-0113-2$.
22. Colombel JF, Sands BE, Rutgeerts P, Sandborn W, Danese $\mathrm{S}$, D'Haens $\mathrm{G}$, et al. The safety of vedolizumab for ulcerative colitis and Crohn's disease. Gut. 2017;66(5):839-851. doi: 10.1136/gutjnl-2015-311079.

23. Hirsch A, Colman RJ, Lang GD, Rubin DT. Successful Treatment of Ulcerative Colitis With Vedolizumab in a Patient With an Infliximab-Associated Psoriasiform Rash. ACG Case Rep J. 2015;2(4):236-8. doi: 10.14309/ crj.2015.70. 